\title{
THE NONEXISTENCE OF EXPANSIVE HOMEOMORPHISMS OF A CLASS OF CONTINUA WHICH CONTAINS ALL DECOMPOSABLE CIRCLE-LIKE CONTINUA
}

\author{
HISAO KATO
}

\begin{abstract}
A homeomorphism $f: X \rightarrow X$ of a compactum $X$ with metric $d$ is expansive if there is $c>0$ such that if $x, y \in X$ and $x \neq y$, then there is an integer $n \in \mathbf{Z}$ such that $d\left(f^{n}(x), f^{n}(y)\right)>c$. It is well-known that $p$ adic solenoids $S_{p}(p \geq 2)$ admit expansive homeomorphisms, each $S_{p}$ is an indecomposable continuum, and $S_{p}$ cannot be embedded into the plane. In case of plane continua, the following interesting problem remains open: For each $1 \leq n \leq 3$, does there exist a plane continuum $X$ so that $X$ admits an expansive homeomorphism and $X$ separates the plane into $n$ components? For the case $n=2$, the typical plane continua are circle-like continua, and every decomposable circle-like continuum can be embedded into the plane. Naturally, one may ask the following question: Does there exist a decomposable circle-like continuum admitting expansive homeomorphisms? In this paper, we prove that a class of continua, which contains all chainable continua, some continuous curves of pseudo-arcs constructed by W. Lewis and all decomposable circle-like continua, admits no expansive homeomorphisms. In particular, any decomposable circle-like continuum admits no expansive homeomorphism. Also, we show that if $f: X \rightarrow X$ is an expansive homeomorphism of a circlelike continuum $X$, then $f$ is itself weakly chaotic in the sense of Devaney.
\end{abstract}

\section{INTRODUCTION}

All spaces considered in this paper are assumed to be separable metric spaces. Maps are continuous functions. By a compactum we mean a nonempty compact metric space. A continuum is a connected compactum. A homeomorphism $f$ : $X \rightarrow X$ of a compactum $X$ with metric $d$ is called expansive ([20], [1] and [2]) if there is $c>0$ such that for any $x, y \in X$ with $x \neq y$, there is an integer $n \in \mathbf{Z}$ such that

$$
d\left(f^{n}(x), f^{n}(y)\right)>c .
$$

A homeomorphism $f: X \rightarrow X$ of a compactum $X$ is continuum-wise expansive [8] if there is $c>0$ such that if $A$ is a nondegenerate subcontinuum of $X$, then there is an integer $n \in \mathbf{Z}$ such that

$$
\operatorname{diam} f^{n}(A)>c,
$$

where $\operatorname{diam} B=\sup \{d(x, y) \mid x, y \in B\}$ for a set $B$. Such a positive number $c$ is called an expansive constant for $f$. Note that each expansive homeomorphism

Received by the editors October 9, 1995 and, in revised form, February 6, 1996.

1991 Mathematics Subject Classification. Primary 54H20, 54F50; Secondary 54E50, 54B20.

Key words and phrases. Expansive homeomorphism, decomposable, chainable, circle-like, the pseudo-arc, pattern, hyperspace. 
is continuum-wise expansive, but the converse assertion is not true. There are many continuum-wise expansive homeomorphisms which are not expansive (e.g., see [8], [9] and [11]). In fact, there are many decomposable circle-like continua admitting continuum-wise expansive homeomorphisms. By the definitions, we see that expansiveness and continuum-wise expansiveness do not depend on the choice of the metric $d$ of $X$. These notions have been extensively studied in the area of topological dynamics, ergodic theory and continuum theory (e.g., see [1], [2], $[7]-[12],[20]$, and [21]).

Let $f: X \rightarrow X$ be a homeomorphism of a compactum $X$. A (nonempty) closed subset $M$ of $X$ is a minimal set of $f$ if $M$ is $f$-invariant, i.e., $f(M)=M$, and for any $x \in M$, the orbit $O(f)=\left\{f^{n}(x) \mid n \in \mathbf{Z}\right\}$ is dense in $M$. Note that every homeomorphism of a compactum has a minimal set. For a point $x \in X$, the $\omega$-limit set $\omega f(x)$ of $x$ is the set

$$
\begin{aligned}
\omega f(x)(=\omega(x))=\{y \in X \mid & \text { there is a sequence } n_{1}<n_{2}<\ldots \\
& \text { of natural numbers such that } \left.\lim _{i \rightarrow \infty} f^{n_{i}}(x)=y\right\} .
\end{aligned}
$$

Similarly, the $\alpha$-limit set $\alpha f(x)(=\alpha(x))$ of $x$ is the set $\omega f^{-1}(x)$.

Let $X$ be a compactum. Let $2^{X}$ be the set of all nonempty closed sets of $X$ and $C(X)$ the set of all nonempty subcontinua of $X$. Suppose that $U_{1}, \ldots, U_{n}$ are nonempty open sets of $X$. Put

$$
\left\langle U_{1}, \ldots, U_{n}\right\rangle=\left\{A \in 2^{X} \mid A \cap U_{i} \neq \phi, A \subset \bigcup_{i=1}^{n} U_{i}\right\}
$$

Then

$$
\begin{aligned}
\beta=\{ & \left\langle U_{1}, U_{2}, \ldots, U_{n}\right\rangle \mid n \geq 1 \text { and } \\
& \left.U_{i}(i \leq n) \text { are nonempty open sets of } X\right\}
\end{aligned}
$$

is a base of $2^{X}$, and it is called the Vietoris topology. Then $2^{X}$ and $C(X)$ are compacta. The spaces $2^{X}$ and $C(X)$ are called the hyperspaces of $X$. For a map $f: X \rightarrow X$, we define a map $f_{*}: 2^{X} \rightarrow 2^{X}$ by $f_{*}(A)=f(A)(=\{f(a) \mid a \in A\})$ for $A \in 2^{X}$. Also, put $C(f)=f_{*} \mid C(X): C(X) \rightarrow C(X)$. Then $X$ is identified with the closed invariant subset of singletons, i.e., degenerate subcontinua.

For the map $C(f): C(X) \rightarrow C(X)$, we shall deal with $\omega(E)=\omega C(f)(E)$ and $\alpha(E)=\omega C(f)^{-1}(E)$ for $E \in C(X)$.

For a homeomorphism $f: X \rightarrow X$, if $Z \subset X$ is a closed invariant subset for $X$, then $Z$ is isolated if for some neighborhood $U$ of $Z$ in $X$ any orbit lying entirely in $U$ is in fact in $Z$, i.e., $Z=\bigcap_{-\infty}^{\infty} f^{n}(U)$. Then $f$ is expansive (resp. continuum-wise expansive) if and only if $X$ is isolated in $2^{X}$ for $f_{*}$ (resp. in $C(X)$ for $C(f)$ ) (see $[1])$.

Let $\mathbf{A}$ and $\mathbf{B}$ be closed $C(f)$-invariant sets in $C(X)$. Then we define the orderings ${ }_{*}<,<_{*}$, and $*_{*}<_{*}$ as follows: Define $\mathbf{A}_{*}<\mathbf{B}$ (resp. $\mathbf{A}<_{*} \mathbf{B}$ ) iff for any $A \in \mathbf{A}$ there is $B \in \mathbf{B}$ (resp. for any $B \in \mathbf{B}$ there is $A \in \mathbf{A}$ ) such that $A \subset B$. Also, define $\mathbf{A}_{*}<_{*} \mathbf{B}$ iff $\mathbf{A}_{*}<\mathbf{B}$ and $\mathbf{A}<_{*} \mathbf{B}$. Example: for $E_{0}, E_{1} \in C(X), E_{0} \subset E_{1}$ implies $\omega\left(E_{0}\right)_{*}<_{*} \omega\left(E_{1}\right)$ and $\alpha\left(E_{0}\right)_{*}<_{*} \alpha\left(E_{1}\right)$. 
For a homeomorphism $f: X \rightarrow X$, we define sets of stable and unstable nondegenerate subcontinua of $X$ as follows (see [9]):

$$
\begin{gathered}
\mathbf{V}^{s}=\{A \mid A \text { is a nondegenerate subcontinuum of } X \text { such that } \\
\left.\mathbf{l i m}_{n \rightarrow \infty} \operatorname{diam} f^{n}(A)=0\right\}, \\
\mathbf{V}^{u}=\{A \mid A \text { is a nondegenerate subcontinuum of } X \text { such that } \\
\left.\lim _{n \rightarrow \infty} \operatorname{diam} f^{-n}(A)=0\right\} .
\end{gathered}
$$

For each $0<\delta<\epsilon$, put

$$
\begin{aligned}
\mathbf{V}^{s}(\delta ; \epsilon) & =\left\{A \in C(X) \mid \operatorname{diam} A \geq \delta, \text { and } \operatorname{diam} f^{n}(A) \leq \epsilon \text { for each } n \geq 0\right\}, \\
\mathbf{V}^{u}(\delta ; \epsilon) & =\left\{A \in C(X) \mid \operatorname{diam} A \geq \delta, \text { and } \operatorname{diam} f^{-n}(A) \leq \epsilon \text { for each } n \geq 0\right\} .
\end{aligned}
$$

Then $\mathbf{V}^{\sigma}(\delta ; \epsilon)(\sigma=u, s)$ is closed in $C(X)$. Note that if $f: X \rightarrow X$ is a continuumwise expansive homeomorphism with an expansive constant $c>0$, then for each $0<\delta<\epsilon<c$ we have $\mathbf{V}^{\sigma}(\delta ; \epsilon) \subset \mathbf{V}^{\sigma}$, and $\mathbf{V}^{\sigma}$ is an $F_{\sigma}$-set in $C(X)$.

A chain $C=\left[C_{1}, C_{2}, \ldots, C_{m}\right]$ of $X$ is a finite collection of open sets of $X$ satisfying the following property:

$$
\mathrm{Cl}\left(C_{i}\right) \cap \mathrm{Cl}\left(C_{j}\right) \neq \phi \text { if and only if }|i-j| \leq 1 .
$$

Each $C_{i}$ is called a link of the chain $C$. Moreover, if for each $i=1, \ldots, m$, $\operatorname{diam}\left(C_{i}\right)<\epsilon$, i.e., $\operatorname{mesh}(C)<\epsilon$, then we say that the chain $C$ is an $\epsilon$-chain. For a chain $C=\left[C_{1}, C_{2}, \ldots, C_{m}\right]$ and two points $p, q \in X$, if $p \in C_{1}$ and $q \in C_{m}$, we say that $C=\left[C_{1}, C_{2}, \ldots, C_{m}\right]$ is a chain from $p$ to $q$. A continuum $X$ is chainable if for any $\epsilon>0$ there is an $\epsilon$-chain covering of $X$.

If $n$ is a natural number, let $I(n)=\{1,2, \ldots, n\}$. A surjective function $f$ : $I(m) \rightarrow I(n)$ is called a pattern provided that $|f(i+1)-f(i)| \leq 1$ for each $i=1, \ldots, m-1$. Let $C=\left[C_{1}, C_{2}, \ldots, C_{n}\right]$ and $D=\left[D_{1}, D_{2}, \ldots, D_{m}\right]$ be chain coverings of $X$ and let $f: I(m) \rightarrow I(n)$ be a pattern. We say that $D$ follows the pattern $f$ in $C$ provided that $D_{i} \subset C_{f(i)}$ for each $i \in I(m)$.

Let $\mathcal{P}$ be a family of compact polyhedra. A continuum $X$ is called a $\mathcal{P}$-like continuum if for any $\epsilon>0$ there is an onto map $g: X \rightarrow P$ such that $P \in \mathcal{P}$ and $\operatorname{diam} g^{-1}(y)<\epsilon$ for each $y \in P$. Note that $X$ is chainable if and only if $X$ is arc-like. A circular chain differs from a chain in that the first and last links intersect. Then a continuum $X$ is circle-like if and only if for any $\epsilon>0$, there is an $\epsilon$-circular chain covering of $X$.

Concerning expansive homeomorphisms, we have the following general problem:

Problem 1.1. What kinds of (plane) continua admit expansive homeomorphisms?

Note that $p$-adic solenoids $S_{p}(p \geq 2)$ are indecomposable circle-like continua admitting expansive homeomorphisms (see [21]), and they cannot be embedded into the plane $R^{2}$. On the other hand, each decomposable circle-like continuum $X$ can be embedded into $R^{2}$, and $R^{2}-X$ has at most 2 components. It is known that for each $n \geq 4$ there is a plane continuum $X$ which is called a Lake of Wada, such that $X$ admits an expansive homeomorphism and $R^{2}-X$ has $n$ components. It is not known whether there exists a plane continuum $X$ such that $X$ admits an expansive homeomorphism and $X$ separates the plane $R^{2}$ into $n$ components $(n \leq 3)$, or not. For the case $n=2$, the typical continua are circle-like continua.

In $[7,8]$, we proved that if $X$ is a tree-like continuum admitting a continuumwise expansive homeomorphism, it must contain an indecomposable subcontinuum. 
Also, in [10], we proved that chainable continua admit no expansive homeomorphisms. Naturally, we are interested in the following problem:

Problem 1.2. Does there exsist a decomposable circle-like continuum admitting an expansive homeomorphism?

In this paper, we prove that some kinds of continua, including all chainable continua, some continuous curves of pseudo-arcs constructed by W. Lewis and all decomposable circle-like continua, admit no expansive homeomorphisms. In particular, any decomposable circle-like continuum admits no expansive homeomorphism. For example, we know that a solenoid of pseudo-arcs and the circle of pseudo-arcs admit no expansive homeomorphisms. Also, we show that if $f: X \rightarrow X$ is an expansive homeomorphism of a circle-like continuum $X$, then $f$ is itself weakly chaotic in the sense of Devaney.

\section{Preliminaries}

A continuum $X$ is decomposable if there are two proper subcontinua $A$ and $B$ of $X$ such that $A \cup B=X$. A continuum $X$ is indecomposable if it is not decomposable. A continuum $X$ is hereditarily indecomposable if each subcontinuum of $X$ is indecomposable. The pseudo-arc $P$ is characterized [4] as a (nondegenerate) hereditarily indecomposable chainable continuum. The pseudo-arc has many remarkable properties in topology and chaotic dynamics (e.g., see [3]-[6] and [13]-[16]). For example, the pseudo-arc $P$ is homogeneous [3], each onto map of the pseudo-arc $P$ is a near homeomorphism [15], and the pseudo-arc $P$ admits chaotic homeomorphisms in the sense of Devaney (see [13]). Also, there is an onto map from the pseudo-arc $P$ to each chainable continuum (see [6] and [14]).

From the proof of $[8$, Proposition 2.3] we have

Lemma 2.1. Let $f: X \rightarrow X$ be a continuum-wise expansive homeomorphism of a compactum $X$ with an expansive constant $c>0$, and let $0<\epsilon<c / 2$. Then there is a positive number $\delta<\epsilon$ such that if $A$ is a subcontinuum of $X$ with $\operatorname{diam} A \leq \delta$ and $\operatorname{diam} f^{m}(A) \geq \epsilon$ for some integer $m \geq 0$ (resp. $m<0$ ), then for each $n \geq m$ and for each $x \in f^{n}(A)$, there is a subcontinuum $B$ of $A$ such that $x \in f^{n}(B)$, $\operatorname{diam} f^{j}(B) \leq$ $\epsilon$ for $0 \leq j \leq n$ and $\operatorname{diam} f^{n}(B)=\delta$ (resp. for each $n \geq-m$ and for each $x \in$ $f^{-n}(A)$, there is a subcontinuum $B$ of $A$ such that $x \in f^{-n}(B), \operatorname{diam} f^{-j}(B) \leq \epsilon$ for $0 \leq j \leq n$, and $\left.\operatorname{diam} f^{-n}(B)=\delta\right)$.

Corollary 2.2. Let $f: X \rightarrow X, c, \epsilon, \delta$ be as in Lemma 2.1.

(a) For every nondegenerate subcontinuum $A$ of $X$ with $\operatorname{diam} A \leq \delta$, exactly one of the two following assertions holds:

1. For all $n \geq 0$, $\operatorname{diam} f^{n}(A) \leq \epsilon$, in which case $A \in \mathbf{V}^{s}$ and $\omega(A) \subset X \subset C(X)$.

2. For $n \geq 0$ sufficiently large, $\operatorname{diam} f^{n}(A) \geq \delta$.

(b) For every subcontinuum $A$, either $\omega(A) \subset X \subset C(X)$ or $\operatorname{diam} E \geq \delta$ for all $E \in \omega(A)$.

For $n \leq 0, \mathbf{V}^{u}$ and $\alpha(A)$, the similar properties are satisfied.

Lemma 2.3 ([8, Corollary 2.4]). Let $f: X \rightarrow X$ be a continuum-wise expansive homeomorphism of a compactum $X$ with $\operatorname{dim} X>0$. Then the following are true.

1. $\mathbf{V}^{u} \neq \phi$ or $\mathbf{V}^{s} \neq \phi$. 
2. If $\delta>0$ is as in the above lemma, then for each $\gamma>0$ there is a natural number $N(\gamma)$ such that if $A$ is a subcontinuum of $X$ with $\operatorname{diam} A \geq \gamma$, then either $\operatorname{diam} f^{n}(A) \geq \delta$ for each $n \geq N(\gamma)$ or $\operatorname{diam} f^{-n}(A) \geq \delta$ for each $n \geq N(\gamma)$.

From the above lemma, we see that $\mathbf{V}^{s} \cap \mathbf{V}^{u}=\phi$ and moreover if $A \in \mathbf{V}^{u}, B \in$ $\mathbf{V}^{s}$, then $\operatorname{dim}(A \cap B) \leq 0$.

Lemma 2.4. Under the same hypothesis as in Lemma 2.3, let $E_{0}, E_{1}$ be nondegenerate subcontinua of $X$ with $E_{1} \in \omega\left(E_{0}\right)$. Then one of the following holds:

1. Every nondegenerate subcontinuum $A_{0}$ of $E_{0}$ with diam $A_{0}<\delta$ lies in $\mathbf{V}^{s}$.

2. There is a subcontinuum $A_{1}$ of $E_{1}$ with $\operatorname{diam} A_{1}=\delta$ lying in $\mathbf{V}^{u}$. Moreover, if $E_{0} \in \mathbf{V}^{u}$, then for any $x \in E_{1}$ there is a subcontinuum $A_{1}$ of $E_{1}$ such that $\operatorname{diam} A_{1}=\delta$ and $x \in A_{1} \in \mathbf{V}^{u}$.

Proof. If the first condition is not true, then there is a subcontinuum $B$ of $E_{0}$ with $0<\gamma=\operatorname{diam} B<\delta$ and a natural number $n$ such that $\operatorname{diam} f^{n}(B)>\epsilon$. Choose a sequence $0=n_{0}<n_{1}<\ldots$, of natural numbers such that $n_{i+1}-n_{i} \geq N(\gamma)$ (see Lemma 2.3) and $\lim _{i \rightarrow \infty} f^{n_{i}}\left(E_{0}\right)=E_{1}$. By using Lemmas 2.1 inductively, we can construct a sequence $B_{0}, B_{1}, \ldots$ of subcontinua with $B_{0}=B$, diam $B_{0}=\gamma<\delta$, $B_{i+1} \subset f^{n_{i+1}-n_{i}}\left(B_{i}\right), \operatorname{diam} B_{i}=\delta(i \geq 1)$, and $\operatorname{diam} f^{-j}\left(B_{i}\right) \leq \epsilon$ for each $0 \leq j \leq$ $n_{i}$. We may assume that $\lim _{i \rightarrow \infty} B_{i}=A_{1}$. Then $A_{1} \in \mathbf{V}^{u}$ and $A_{1} \subset E_{1}$.

Moreover, suppose that $E_{0} \in \mathbf{V}^{u}$. For any $x \in E_{1}$, we choose a sequence $x_{0}, x_{1}, \ldots$ of points such that $x_{i} \in f^{n_{i}}\left(E_{0}\right)$ and $\lim _{i \rightarrow \infty} x_{i}=x$. Choose a subcontinuum $B$ of $E_{0}$ such that $x_{0} \in B$ and $\operatorname{diam} B=\gamma<\delta$. By Lemma 2.1, we can choose a sequence $B_{0}, B_{1}, \ldots$ satisfying the above conditions with $x_{i} \in B_{i}$ for each $i$. Then $x \in A_{1} \in \mathbf{V}^{u}$.

Corollary 2.5. Under the same hypothesis as in Lemma 2.3, let $\mathbf{A}$ be a minimal set of $C(f)$. Assume that there is a nondegenerate subcontinuum $A \in \mathbf{A}$.

(a) For all $A \in \mathbf{A}$, $\operatorname{diam} A \geq \delta$.

(b) Exactly one of the three following conditions holds for $\mathbf{A}$ :

1. For all $A \in \mathbf{A}$ and all subcontinua $B$ of $A$ with $\operatorname{diam} B<\delta, B \in \mathbf{V}^{s}$.

2. For all $A \in \mathbf{A}$ and all subcontinua $B$ of $A$ with $\operatorname{diam} B<\delta, B \in \mathbf{V}^{u}$.

3. For all $A \in \mathbf{A}$ there are subcontinua $B_{0}, B_{1}$ of $A$ with $\operatorname{diam} B_{0}=\operatorname{diam} B_{1}$ $=\delta$ and $B_{0} \in \mathbf{V}^{s}, B_{1} \in \mathbf{V}^{u}$.

(c) If $A \in \mathbf{A}$ and $B$ is a nondegenerate subcontinuum of $A$ with $B \notin \mathbf{V}^{s}$, then $\operatorname{diam} E \geq \delta$ for each $E \in \omega(B), \omega(B)_{*}<_{*} \mathbf{A}$, and if $\mathbf{A}_{0}$ is a minimal set in $\omega(B)$, then $\mathbf{A}_{0 *}<_{*} \mathbf{A}$ as well.

The following propositions are used in the sequel.

Proposition 2.6. Let $f: X \rightarrow X$ be a continuum-wise expansive homeomorphism of a compactum $X$ with $\operatorname{dim} X>0$. Suppose that $\mathbf{B}$ is a $C(f)$-invariant set such that some element of $\mathbf{B}$ is nondegenerate. Then there exists a minimal set $\mathbf{A}_{*}<\mathbf{B}$ of $C(f)$ such that each element of $\mathbf{A}$ is nondegenerate, and such that for each $A \in \mathbf{A}$ and each nondegenerate subcontinuum $B$ of $A$ either $B \in \mathbf{V}^{s}$ or $\mathbf{A} \subset \omega(B)$, and either $B \in \mathbf{V}^{u}$ or $\mathbf{A} \subset \alpha(B)$.

Proof. For pairs $(A, \mathbf{A})$ such that $\mathbf{A}$ is minimal, $\mathbf{A}_{*}<\mathbf{B}, A \in \mathbf{A}$ and $A$ is a nondegenerate subcontinuum, we consider the order by inclusion of the $A$ 's. By Corollary 2.2,(b), there exists such a pair. If $\left\{\left(A_{\alpha}, \mathbf{A}_{\alpha}\right)\right\}$ is a totally ordered family, 
then $B=\bigcap_{\alpha} A_{\alpha}$ is a nondegenerate subcontinuum and so either $\omega(B)$ or $\alpha(B)$ contains a minimal subset $\mathbf{A}$ such that its elements are nondegenerate subcontinua and $\mathbf{A}_{*}<_{*} \mathbf{A}_{\alpha}$ for all $\alpha$. For each $\alpha$ choose $B_{\alpha} \subset A_{\alpha}$ with $B_{\alpha} \in \mathbf{A}$. Then any limit point $A$ of the net $\left\{B_{\alpha}\right\}$ is an element of $\mathbf{A}$ contained in all the $A_{\alpha}$ 's. So Zorn's lemma applies to the pairs. If $(\tilde{A}, \mathbf{A})$ is minimal with respect to this ordering, then A satisfies the conclusion. In fact, if $A \in \mathbf{A}$ and $B$ is a nondegenerate subcontinuum of $A$ not in $\mathbf{V}^{s}$, then $\omega(B){ }_{*}<_{*} \mathbf{A}$ and $\mathbf{A}_{0}{ }_{*}<_{*} \mathbf{A}$ for any minimal subset $\mathbf{A}_{0}$ of $\omega(B)$. Then there is $A_{0} \in \mathbf{A}_{0}$ such that $A_{0} \subset \tilde{A}$, and so by minimality we see that $A_{0}=\tilde{A}$ and so $\mathbf{A}=\omega\left(A_{0}\right)=\mathbf{A}_{0} \subset \omega(B)$.

This completes the proof.

Proposition 2.7. Under the same assumption as in the above proposition, the minimal set $\mathbf{A}$ satisfies one of the following conditions:

1. If some $A_{0} \in \mathbf{A}$ contains an element of $\mathbf{V}^{u}$, then for any $x \in A \in \mathbf{A}$, there is a nondegenerate subcontinuum $A_{x}$ of $A$ such that $x \in A_{x} \in \mathbf{V}^{u}$, and if $A^{\prime}$ is a nondegenerate subcontinuum of $A \in \mathbf{A}$ with $A^{\prime} \notin \mathbf{V}^{s}$, then for each $H \in \mathbf{A}$ there is a sequence $n_{1}<n_{2}<\ldots$ of natural numbers such that

$$
\lim _{i \rightarrow \infty} f^{n_{i}}(A)=\lim _{i \rightarrow \infty} f^{n_{i}}\left(A^{\prime}\right)=H .
$$

2. If some $A_{0} \in \mathbf{A}$ contains an element of $\mathbf{V}^{s}$, then for any $x \in A \in \mathbf{A}$, for any $x \in A \in \mathbf{A}$, there is a nondegenerate subcontinuum $A_{x}$ of $A$ such that $x \in A_{x} \in \mathbf{V}^{s}$, and if $A^{\prime}$ is a any nondegenerate subcontinuum of $A \in \mathbf{A}$ with $A^{\prime} \notin \mathbf{V}^{u}$, then for each $H \in \mathbf{A}$ there is a sequence $n_{1}<n_{2}<\ldots$ of natural numbers such that

$$
\lim _{i \rightarrow \infty} f^{-n_{i}}(A)=\lim _{i \rightarrow \infty} f^{-n_{i}}\left(A^{\prime}\right)=H .
$$

Proof. We shall show the first case. Let $B \in \mathbf{V}^{u}$ and $B \subset A_{0} \in \mathbf{A}$. By the above proposition, we see that $\mathbf{A} \subset \omega(B)$. By Lemma 2.4, we see that for any $x \in A \in \mathbf{A}$, there is $A_{x} \in \mathbf{V}^{u}$ such that $x \in A_{x} \subset A$. Since $\mathbf{A}$ is closed in $C(X)$, A contains an maximal element in order by inclusion. In fact, for a Whitney map $\mu: C(X) \rightarrow[0,1]$ (see [18]), we can choose an element $T$ of $\mathbf{A}$ such that $\mu(T)=\max \{\mu(E) \mid E \in \mathbf{A}\}$. Then $T$ is a maximal element of $\mathbf{A}$. Suppose that $A^{\prime}$ is a nondegenerate subcontinuum of $A \in \mathbf{A}$ with $A^{\prime} \notin \mathbf{V}^{s}$. Let $H \in \mathbf{A}$. Since $\omega\left(A^{\prime}\right) \supset$ A (see Proposition 2.6), $T \in \omega\left(A^{\prime}\right)$. Hence there is a sequence $i_{1}<i_{2}<\ldots$ of natural numbers such that $\lim _{k \rightarrow \infty} f^{i_{k}}\left(A^{\prime}\right)=T$. We may assume that $\left\{f^{i_{k}}(A)\right\}_{k=1}^{\infty}$ is convergent. Since $T$ is maximal in $\mathbf{A}$, we see that $\lim _{k \rightarrow \infty} f^{i_{k}}(A)=T$. Since $\mathbf{A}$ is minimal, $H \in \omega(T)$. Then we can choose a sequence $n_{1}<n_{2}<\ldots$ of natural numbers such that

$$
\lim _{i \rightarrow \infty} f^{n_{i}}\left(A^{\prime}\right)=\lim _{i \rightarrow \infty} f^{n_{i}}(A)=H .
$$

This completes the proof.

Let $f: X \rightarrow X$ be a continuum-wise expansive homeomorphism of a compactum $X$ with $\operatorname{dim} X>0$. Note that every minimal set of $f$ is 0 -dimensional (see [8, Theorem 5.2]). Consider the following sets (see [12]):

1. $\mathcal{I}(f)=\left\{A \in 2^{X} \mid A\right.$ is $f$-invariant $\}$.

2. $\mathcal{I}^{+}(f)=\{A \in \mathcal{I}(f) \mid \operatorname{dim} A>0\}$.

3. $\mathcal{D}(f)$ is the set of all minimal elements of $\mathcal{I}^{+}(f)$ in the order by inclusion. 
Note that $\mathcal{D}(f) \neq \phi$ (see [12, Proposition 2.4]) and if $Y \in \mathcal{D}(f)$, then $f_{Y}=f \mid Y$ : $Y \rightarrow Y$ is weakly chaotic in the sense of Devaney, i.e., $f_{Y}$ has sensitive dependence on initial conditions, $f_{Y}$ is topologically transitive and the union of all minimal sets of $f_{Y}$ is dense in $Y$ ( $\left[12\right.$, Theorem 2.7]), i.e., the min-center of $f_{Y}$ is $Y$ (see [1, p. 70]).

Proposition 2.8. Let $f: X \rightarrow X$ be a continuum-wise expansive homeomorphism of a compactum $X$ with $\operatorname{dim} X>0$. If $Y \in \mathcal{D}(f)$, then there is a minimal set A of $C(f)$ satisfying one of the conditions (1) and (2) as in Proposition 2.7 and $\bigcup\{A \mid A \in \mathbf{A}\}=Y$.

Proof. Consider the map $f \mid Y: Y \rightarrow Y$. Then there is a minimal set $\mathbf{A}$ of $C(f \mid Y)$ as in Proposition 2.7. Put $Y^{\prime}=\bigcup\{A \mid A \in \mathbf{A}\}$. Then $Y^{\prime}$ is $f$-invariant and $\operatorname{dim} Y^{\prime}>0$. Hence $Y=Y^{\prime}$.

The following lemma follows from [3, Theorem 6] (see also [15, Lemmas 2 and 1.1]).

Lemma 2.9. Let $P$ be the pseudo-arc. Let $C=\left[C_{1}, C_{2}, \ldots, C_{n}\right]$ be a chain covering of $P$ and $f: I(m) \rightarrow I(n)$ a pattern with $f(1)=1$. Let $p \in C_{1}$. Then there is a chain covering $D=\left[D_{1}, D_{2}, \ldots, D_{m}\right]$ such that $D$ refines the chain $C, p \in D_{1}$ and $D$ follows the pattern $f$ in $C$.

The following lemma is a simple modification of the uniformization theorem of Mioduszewski (see [17] and [19]). For completeness, we give the proof.

Lemma 2.10. Let $I=[0,1]$ be the unit interval. Suppose that $f, g: I \rightarrow I$ are piecewise linear onto maps. If $f(0)=g(0)=0$, then there are onto maps $a, b: I \rightarrow I$ such that $f \cdot a=g \cdot b$ and $a(0)=b(0)=0$.

Proof. Let $\psi: I^{2} \rightarrow R$ be the map defined by $\psi(x, y)=f(x)-g(y)$. Note that $I^{2}$ is unicoherent (i.e., if $A$ and $B$ are coutinua with $A \cup B=I^{2}$, then $A \cap B$ is connected). In [17], Mioduszewski proved that there is a component $K$ of $\psi^{-1}(0)$ such that $K$ meets all four sides of $I^{2}$ (see also [19]). Note that each component of $\psi^{-1}(0)$ is a polyhedron. Let $L$ be a component of $\psi^{-1}(0)$ containing the point $p=(0,0) \in I^{2}$. Suppose, on the contrary, that $L \cap(I \times\{1\} \cup\{1\} \times I)=\phi$. Then there is an arc $\alpha: I \rightarrow I^{2}$ such that $\alpha(0)=\left(x_{1}, 0\right) \in I \times\{0\}, \alpha(1)=\left(0, y_{1}\right) \in\{0\} \times I$, and $\psi^{-1}(0) \cap \alpha(I)=\phi$. Note that $g(0)=0<f\left(x_{1}\right)$ and $g\left(y_{1}\right)>f(0)$. Hence we can see that there is a point $q=\left(q_{1}, q_{2}\right) \in \alpha(I)$ such that $f\left(q_{1}\right)=g\left(q_{2}\right)$, which implies that $q \in \phi^{-1}(0)$. This is a contradiction. Hence $K$ contains $L$. By using this fact, we can choose desired maps $a, b: I \rightarrow I$.

\section{THE NONEXISTENCE OF EXPANSIVE HOMEOMORPHISMS OF CERTAIN CONTINUA}

The following is the main theorem in this paper.

Theorem 3.1. Let $f: X \rightarrow X$ be a homeomorphism of a compactum $X$. If there is a minimal set $\mathbf{A}$ of $C(f)$ such that some element $A$ of $\mathbf{A}$ is a (nondegenerate) chainable continuum, then $f$ is not expansive.

Proof. Suppose, on the contrary, that $f$ is expansive. Replace $\mathbf{A}$ if necessary by an $\mathbf{A}_{0 *}<\mathbf{A}$ which satisfies the condition (1) of Proposition 2.7. Since every subcontinuum of a chainable continuum is also chainable, we may assume that $\mathbf{A}$ satisfies the conditions of Proposition 2.7,(1). 
Let $c>0$ be an expansive constant for $f$ and $c / 2>\epsilon>0$. Now, we shall prove the following property

For any $0<\tau<\epsilon$ there are two points $x, y$ of $X$ and a natural number $n(\tau)$

$$
\begin{gathered}
\text { such that } d(x, y) \leq \tau, d\left(f^{n(\tau)}(x), f^{n(\tau)}(y)\right) \leq \tau, \text { and } \\
\epsilon \leq \sup \left\{d\left(f^{j}(x), f^{j}(y)\right) \mid 0 \leq j \leq n(\tau)\right\} \leq 2 \epsilon .
\end{gathered}
$$

Let $A \in \mathbf{A}$ be a chainable continuum. Since $A$ is chainable, there is a $\tau / 4$-chain $C=\left[C_{1}, C_{2}, \ldots, C_{r}\right]$ in $X$ which is an open covering of $A$. We can choose a subcontinuum $B_{1}$ of $A$ such that $B_{1} \in \mathbf{V}^{u}(\tau ; \epsilon)$ (see (1) of Proposition 2.7), and we may assume that $\operatorname{diam}\left(B_{1}\right)=\tau$. Since $B_{1} \in \mathbf{V}^{u}$ and $f$ is expansive, we can choose a natural number $N_{1}$ such that if $x, y \in B_{1}$ and $d(x, y) \geq \tau / 4$, then

$$
\sup \left\{d\left(f^{i}(x), f^{i}(y)\right) \mid 0 \leq i \leq N_{1}\right\}>2 \epsilon .
$$

Choose a subcontinuum $B_{2}$ of $B_{1}$ such that diam $B_{2}=\tau / 2$. By the assumption, there is a sequence $n_{1}<n_{2}<\ldots$ of natural numbers such that $\lim _{i \rightarrow \infty} f^{n_{i}}\left(B_{2}\right)=$ $\lim _{i \rightarrow \infty} f^{n_{i}}(A)=A$ (see Proposition 2.7). Hence, we can choose a natural number $N>N_{1}$ such that $f^{N}\left(B_{1}\right), f^{N}\left(B_{2}\right) \in\left\langle C_{1}, \ldots, C_{r}\right\rangle$. Choose a point $e \in B_{2}$ such that $f^{N}(e) \in C_{1}$. Since $B_{1}, B_{2}$ are chainable, by [6] or [14] there are onto maps $\psi_{k}: P \rightarrow B_{k}(k=1,2)$ from the pseudo-arc $P$ onto $B_{k}$. Let $p \in P$. Since $P$ is homogeneous [3], we may assume that $\psi_{k}(p)=e$ for each $k=1,2$. Choose a chain covering $D=\left[D_{1}, \ldots, D_{s}\right]$ of $P$ such that its mesh is sufficiently small. We may assume that if $x, y \in D_{i} \cup D_{i+1}$, then

$$
\sup \left\{d\left(f^{j}\left(\psi_{k}(x)\right), f^{j}\left(\psi_{k}(y)\right)\right) \mid 0 \leq j \leq N\right\}<\epsilon / 2
$$

for each $k=1,2$. We may assume that $p \in D_{1}$ (see the proof of [3, Theorem 13]). Also we may assume that $D$ is a refinement of the chains $C^{k}=\left(f^{N} \cdot \psi_{k}\right)^{-1}(C)(k=$ $1,2)$. Let $f_{k}: I(s) \rightarrow I(r)(k=1,2)$ be patterns such that $D$ follows the patterns $f_{k}$ in $C^{k}(k=1,2)$. Then the patterns $f_{k}(k=1,2)$ induce maps $f_{k}: N(D)=$ $N\left(\left\{D_{1}, \ldots, D_{s}\right\}\right) \rightarrow N(C)=N\left(\left\{C_{1}, \ldots, C_{r}\right\}\right)$ which are natural simplicial maps from the nerve $N(D)$ of $D$ to $N(C)$ of $C$ with $f_{k}\left(D_{j}\right)=C_{f_{k}(j)}$ for each $j$.

Since the above nerves are arcs, we can consider that $f_{k}$ is a map from the unit interval $I$ onto $I$ such that $f_{k}(0)=0(k=1,2)$. By Lemma 2.10, there are onto maps $g_{k}: I \rightarrow I$ such that $f_{1} \cdot g_{1}=f_{2} \cdot g_{2}$ and $g_{k}(0)=0$.

By using $g_{k}(k=1,2)$, we obtain patterns $g_{k}: I(l) \rightarrow I(s)$ satisfying the inequality $\left|f_{1} g_{1}(j)-f_{2} g_{2}(j)\right| \leq 1$ for each $j=1,2, \ldots, l$. By Lemma 2.9 , we can choose chain coverings $E=\left[E_{1}, E_{2}, \ldots, E_{l}\right]$ and $F=\left[F_{1}, F_{2}, \ldots, F_{l}\right]$ of $P$ such that $E$ follows the pattern $g_{1}$ in $D$ and $F$ follows the pattern $g_{2}$ in $D$. We may assume that $p \in E_{1} \cap F_{1}$.

Choose points $a_{1}, \ldots, a_{l}, b_{1}, \ldots, b_{l}$ of $P$ beginning with $p=a_{1}=b_{1}$ and such that $a_{j} \in E_{j}, b_{j} \in F_{j}$. Note that

$$
d\left(f^{N}\left(\psi_{1}\left(a_{j}\right)\right), f^{N}\left(\psi_{2}\left(b_{j}\right)\right)\right) \leq \tau .
$$

For each $i=1,2, \ldots, l$, put

$$
r_{i}=\sup \left\{d\left(f^{j}\left(\psi_{1}\left(a_{i}\right)\right), f^{j}\left(\psi_{2}\left(b_{i}\right)\right)\right) \mid 0 \leq j \leq N\right\} .
$$

Since the chain cover $D$ is sufficiently small (see (3.1.2)), we may assume that

$$
\left|r_{i}-r_{i+1}\right|<\epsilon \text {. }
$$


Note that $r_{1}=0$. Since $\psi_{1}$ is surjective, there is a point $a_{u}(u \leq l)$ such that $d\left(\psi_{1}\left(a_{u}\right), B_{2}\right) \geq \tau / 4$, and hence $d\left(\psi_{1}\left(a_{u}\right), \psi_{2}\left(b_{u}\right)\right) \geq \tau / 4$. Thus $r_{u}>2 \epsilon$. Then we can choose $i \leq u$ such that $\epsilon \leq r_{i} \leq 2 \epsilon$. The two points $a_{i}, b_{i}$ satisfy the condition (3.1.1).

Let $\left\{\epsilon_{i}\right\}_{i=1}^{\infty}$ be a sequence of positive numbers such that $\lim _{i \rightarrow \infty} \epsilon_{i}=0$. By the condition (3.1.1), there are two points $x_{i}, y_{i} \in X$ and a natural number $n(i)$ such that

$$
d\left(x_{i}, y_{i}\right)<\epsilon_{i}, \quad d\left(f^{n(i)}\left(x_{i}\right), f^{n(i)}\left(y_{i}\right)\right)<\epsilon_{i}
$$

and

$$
\epsilon \leq \sup \left\{d\left(f^{j}\left(x_{i}\right), f^{j}\left(y_{i}\right)\right) \mid 0 \leq j \leq n(i)\right\} \leq 2 \epsilon .
$$

Choose $0<m(i)<n(i)$ such that $d\left(f^{m(i)}\left(x_{i}\right), f^{m(i)}\left(y_{i}\right)\right) \geq \epsilon$. We may assume that $\left\{f^{m(i)}\left(x_{i}\right)\right\}$ and $\left\{f^{m(i)}\left(y_{i}\right)\right\}$ are convergent to $x_{0}$ and $y_{0}$, respectively. Note that

$$
\lim _{i \rightarrow \infty}(n(i)-m(i))=\infty=\lim _{i \rightarrow \infty} m(i) .
$$

Then $x_{0} \neq y_{0}$ and $d\left(f^{n}\left(x_{0}\right), f^{n}\left(y_{0}\right)\right) \leq 2 \epsilon<c$ for all $n \in \mathbf{Z}$. This is a contradiction.

Corollary 3.2. If $X$ is a decomposable circle-like continuum, then $X$ admits no expansive homeomorphism.

Proof. Suppose, on the contrary, that there is an expansive homeomorphism $f$ : $X \rightarrow X$. Since $X$ is decomposable, there are two proper nonempty subcontinua $A, B$ of $X$ such that $A \cup B=X$. Since $X$ is circle-like, $A$ and $B$ are chainable. Note that $A \cap B$ has at most 2 components [5, Theorem 5]. By [11, Theorem 3.6], there is a $\sigma$-chaotic continuum $C$ of $f$. We may assume that $\sigma=u$. Then $C$ is indecomposable (see [11, Corollary 5.3]) and is a proper subcontinuum of $X$. Note that $f^{n}(C) \cap A$ and $f^{n}(C) \cap B$ have at most 2 components. Since $f^{n}(C)$ is indecomposable, we can easily see that for each $n=0,1, \ldots, f^{n}(C) \subset A$ or $f^{n}(C) \subset B$. Hence we see that there is a minimal set $\mathbf{A}$ of $C(f)$ satisfying the condition of Theorem 3.1. By Theorem 3.1, $f$ is not expansive.

Corollary 3.3. Let $f: X \rightarrow X$ be a homeomorphism of a compactum $X$. Suppose that there are maps $\psi: X \rightarrow Y$ and $g: Y \rightarrow Y$ such that $\psi \cdot f=g \cdot \psi$ and for each $y \in Y \psi^{-1}(y)$ is a (nondegenerate) chainable continuum. Then $f$ is not expansive.

Proof. Let $y_{0} \in Y$. By Corollary 2.2, we may assume each element of $\omega\left(\psi^{-1}\left(y_{0}\right)\right)$ is nondegenerate. Since $\psi \cdot f=g \cdot \psi$ and the collection $\left\{\psi^{-1}(y) \mid y \in Y\right\}$ is an upper semi-continuous decomposition of $X$, each element of $\omega\left(\psi^{-1}\left(y_{0}\right)\right)$ is contained in some $\psi^{-1}(y)$, and hence it is chainable.

Take a minimal set $\mathbf{A}$ of $\omega\left(\psi^{-1}\left(y_{0}\right)\right)$. Then each element of $\mathbf{A}$ is a chainable continuum. By Theorem 3.1, $f$ is not expansive.

Corollary 3.4. Let $f: X \rightarrow X$ be an expansive homeomorphism of a circle-like continuum $X$, and let $\delta>0$ be a positive number as in Lemma 2.1. Then one of the following conditions is satisfied:

(i) If $A \in C(X)$ and $0<\operatorname{diam} A<\delta$, then $A \in \mathbf{V}^{u}$, and if $B$ is a nondegenerate subcontinuum of $X$, then $X \in \omega(B)$.

(ii) If $A \in C(X)$ and $0<\operatorname{diam} A<\delta$, then $A \in \mathbf{V}^{s}$, and if $B$ is a nondegenerate subcontinuum of $X$, then $X \in \alpha(B)$. 
Proof. By Lemma 2.3, we may assume that $\mathbf{V}^{u} \neq \phi$. Let $A \in \mathbf{V}^{u}$. Suppose, on the contrary, that $\omega(A)$ does not contain $X$. Then we obtain a minimal set $\mathbf{A} \subset \omega(A)$ of $C(f)$ satisfying the condition of Theorem 3.1. Hence $f$ is not expansive, which is a contradiction. Since $X \in \omega(A)$, by Lemma 2.4 we see that for each $x \in X$ there is $x \in A_{x} \in \mathbf{V}^{u}(\delta ; \epsilon)$, where $\delta, \epsilon$ are as in Lemma 2.1. Suppose, on the contrary, that $\mathbf{V}^{s} \neq \phi$. Then we see also that for each $x \in X$ there is $x \in B_{x} \in \mathbf{V}^{s}(\delta ; \epsilon)$. Since $f$ is expansive, we know that $A_{x} \cap B_{x}=\{x\}$. Choose $A_{x}$ and two points $y, z \in A_{x}$ such that $x, y$ and $z$ are different. Then there are three subcontinua $B_{x}, B_{y}, B_{z}$ such that their diameters are small and $B_{x}, B_{y}$ and $B_{z}$ are mutually disjoint. Then $T=A_{x} \cup B_{x} \cup B_{y} \cup B_{z}$ is a triod. Since $X$ is atriodic, this is a contradiction. Hence $\mathbf{V}^{s}=\phi$. Let $A$ be a nondegenerate subcontinuum of $X$ with $\operatorname{diam} A=\gamma<\delta$. Suppose, on the contrary, that $\sup \left\{\operatorname{diam} f^{-n}(A) \mid n \geq 0\right\} \geq \epsilon$. By using Lemmas 2.1 and 2.3 inductively, we have a sequence $n_{1}<n_{2}<\ldots$ of natural numbers and a sequence $B_{1}, B_{2}, \ldots$ of subcontinua such that $B_{i} \subset f^{-n_{i}}(A)$, diam $B_{i}=\delta$ and $\operatorname{diam} f^{j}\left(B_{i}\right) \leq \epsilon$ for each $0 \leq j \leq n_{i}$. We may assume that $\lim _{i \rightarrow \infty} B_{i}=B \in \mathbf{V}^{s}$. This is a contradiction. Hence we see that $A \in \mathbf{V}^{u}$. Clearly, if $B$ is a nondegenerate subcontinuum, then $X \in \omega(B)$, because $B \notin \mathbf{V}^{s}$.

Corollary 3.5. If $f: X \rightarrow X$ is an expansive homeomorphism of a circle-like continuum $X$, then $f$ is itself weakly chaotic in the sense of Devaney.

Proof. Consider the set $\mathcal{D}(f) \neq \phi$. Let $Y \in \mathcal{D}(f)$. Since $\operatorname{dim} Y>0, Y$ contains a nondegenerate subcontinuum. By Corollary 3.4, we see that $Y=X$. Hence $f$ is weakly chaotic in the sense of Devaney.

Remark. In [16], Lewis showed that, for every 1-dimensional continuum $M$ there exists a 1-dimensional continuum $\hat{M}$ such that $\hat{M}$ has a continuous decomposition $\psi: \hat{M} \rightarrow M$ into pseudo-arcs such that the decomposition space is homeomorphic to $M$ and the decomposition elements are all terminal continua in $\hat{M}$, i.e., every subcontinuum of $\hat{M}$ either is contained in a single decomposition element or is a union of decomposition elements. More generally, let $\tilde{N}$ be a compactum that has an upper semi-continuous decomposition $\varphi$ into indecomposable chainable continua such that the decomposition elements are all terminal, and let $N$ be the decomposition space. Moreover, if each proper subcontinuum of $N$ is decomposable, then for any homeomorphism $\tilde{h}: \tilde{N} \rightarrow \tilde{N}$ there is a homeomorphism $h: N \rightarrow N$ such that $\varphi \cdot \tilde{h}=h \cdot \varphi$. By Corollary $3.3, \tilde{N}$ admits no expansive homeomorphism. The typical continua are solenoids of pseudo-arcs and hence they admit no expansive homeomorphisms.

Problem 3.6. Does there exist an indecomposable plane circle-like continuum which admits an expansive homeomorphism? In particular, does the pseudo-circle admit an expansive homeomorphism?

Problem 3.7. Does there exist a hereditarily indecomposable continuum which admits an expansive homeomorphism?

The author wishes to thank the referee for very helpful suggestions and comments, in particular, the proof of Proposition 2.6. 


\section{REFERENCES}

1. E. Akin, The General Topology of Dynamical Systems, Amer. Math. Soc., Providence, 1993. MR 94f: 58041

2. N. Aoki, Topological dynamics, in: Topics in general topology (eds, K. Morita and J. Nagata), North-Holland, Amsterdam, (1989), 625-740. MR 91m:58120

3. R. H. Bing, A homogeneous indecomposable plane continuum, Duke Math. J., 15 (1948), 729-742. MR 10:261a

4. R. H. Bing, Concerning hereditarily indecomposable continua, Pacific J. Math., 1 (1951), 43-51. MR 13:265b

5. C. E. Burgess, Chainable continua and indecomposability, Pacific J. Math., 9 (1959), 653-659. MR 22:1867

6. L. Fearnley, Characterizations of the continuous images of the pseudo-arc, Trans. Amer. Math. Soc., 111 (1964), 380-399. MR 29:596

7. H. Kato, Expansive homeomorphisms in continuum theory, Topology Appl., 45 (1992), 223243. MR 93j:54023

8. Continuum-wise expansive homeomorphisms, Canad. J. Math., 45 (1993), 576-598. MR 94k:54065

9. Chaotic continua of (continuum-wise) expansive homeomorphisms and chaos in the sense of Li and Yorke, Fund. Math., 145 (1994), 261-279. MR 95i:54049

10. The nonexistence of expansive homeomorphisms of chainable continua, Fund. Math., 149 (1996), 119-126. CMP 96:09

11. Chaos of continuum-wise expansive homeomorphisms and dynamical properties of sensitive maps of graphs, Pacific J. Math., 175 (1996), 93-116. CMP 97:04

12. Minimal sets and chaos in the sense of Devaney on continuum-wise expansive homeomorphisms, Lecture Notes in Pure and Applied Mathematics, 170 (1995), 265-274. MR 96c:54065

13. J. Kennedy, The construction of chaotic homeomorphisms on chainable continua, Topology Appl., 43 (1992), 91-116. MR 93b:54040

14. A. Lelek, On weakly chainable continua, Fund. Math., 51 (1962), 271-282. MR 26:742

15. W. Lewis, Most maps of the pseudo-arc are homeomorphisms, Proc. Amer. Math. Soc., 91 (1984), 147-154. MR 85g:54025

16. W. Lewis, Continuous curves of pseudo-arcs, Houston J. Math., 11 (1985), 91-99. MR 86e: 54038

17. J. Mioduszewski, On a quasi-ordering in the class of continuous mappings of the closed interval onto itself, Colloq. Math., 9 (1962), 233-240. MR 26:741

18. S. B. Nadler, Jr., Hyperspaces of sets, Pure and Appl. Math., 49 (Dekker, New York, 1978). MR 58:18330

19. L. G. Oversteegen and E. D. Tymchatyn, On span and weakly chainable continua, Fund. Math., 122 (1984), 159-174. MR 85m:54034

20. W. Utz, Unstable homeomorphisms, Proc. Amer. Math. Soc., 1 (1950), 769-774. MR 12:344b

21. R. F. Williams, A note on unstable homeomorphisms, Proc. Amer. Math. Soc., 6 (1955), 308-309. MR 16:846d

Institute of Mathematics, University of Tsukuba, Ibaraki 305, Japan

E-mail address: hisakato@sakura.cc.tsukuba.ac.jp 